Supplement of Atmos. Chem. Phys., 21, 10413-10438, 2021

https://doi.org/10.5194/acp-21-10413-2021-supplement

(C) Author(s) 2021. CC BY 4.0 License.

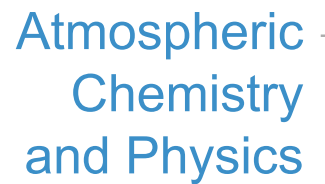

(c) (i)

and Physics

Supplement of

\title{
Present and future aerosol impacts on Arctic climate change in the GISS-E2.1 Earth system model
}

\section{Ulas Im et al.}

Correspondence to: Ulas Im (ulas@envs.au.dk)

The copyright of individual parts of the supplement might differ from the article licence. 
Measurement Techniques

\section{Black carbon}

Measurements of elemental carbon (EC, which is a "type" of BC) uses a thermal/ or thermaloptical method (Chow et al, 1993) after collecting particulate matter on a Quartz filter for the EMEP, IMPROVE (including Fairbanks), and CABM datasets (Torseth et al, 2012; EMEP manual, 2014; Huang et al, 2006; Sharma et al, 2017; Huang et al, 2020). This method determines the total carbon in the particulate material and then splits this measurement into OC and EC based on an optical correction via thermal-optical protocol or only using stepwise temperature to separate OC from EC (Huang et al., 2020). These are reported for $\mathrm{PM}_{2.5}$ and $\mathrm{PM}_{10}$ in the EMEP dataset, and for total suspended particles (TSP) in the Alert (CABM) dataset for the years 2005-2011. From 2011 to present, the Alert measurements of EC are for PM $\mathrm{P}_{1}$. Alert sampling frequency is a weekly integration. The overall uncertainty for Alert EC measurements is approximately upto $30 \%$, and the IMPROVE EC measurements have $0.002 \mu \mathrm{g} \mathrm{C} \mathrm{m} \mathrm{method}^{-3}$ methe detection limit.

Measurements of equivalent black carbon (eBC, which is another "type" of BC) can be done via aethalometer (e.g. complementary measurements at Alert and at Zeppelin), or via particle soot absorption photometer (PSAP, Bond et al, 1999) at Gruvebadet, Zeppelin, and at Utqiagvik (Barrow), and these are for $\mathrm{PM}_{1}$. Both use the light absorption method where the change in light transmission through the filter over time is related to the concentration of eBC. These eBC measurements from different instruments may be systematically offset unless scaled by a third type of measurement (e.g., Continuous Soot Monitoring System, COSMOS; Kondo et al, 2011). While each of these $\mathrm{BC}$ measurement methods are different, and none of them fully represent $\mathrm{BC}$, they have been shown to provide a range of the "true" BC concentrations, and typically agree with each other within a factor of two (2021 AMAP SLCF report, chapter 4).

\section{Sulfate}

For the IMPROVE and CABM networks, water soluble inorganic ions, like SO42-, are measured with ion chromatography. The particulates are caught on nylon filters, then the samples are dissolved in deionized water, separated by ion chromatography and detected by changes in conductivity (Harris, 2003). For EMEP $\mathrm{SO}_{4}{ }^{2-}$ measurements are made daily, particulate matter is collected for 24 hours on Teflon filters kept at ambient conditions. The samples are prepared via water extraction after ultrasonic treatment. The reported concentrations are blank corrected, and the method has a detection limit of 0.01-0.02 $\mu \mathrm{g} \mathrm{S} \mathrm{m}{ }^{-3}$. At Gruvebadet lab, $\mathrm{SO}_{4}{ }^{2-}$ is by TECORA SKYPOST sampler, with a $\mathrm{PM}_{10}$ particle size cutoff. FTFE filters. These $\mathrm{SO}_{4}{ }^{2-}$ measurements have up to $20 \%$ analytical uncertainty (2021 AMAP SLCF report, chapter 4). 


\section{Organic carbon}

As mentioned above, $\mathrm{OC}$ is also measured via a thermal/or thermal- optical method (Chow et al, 1993; Huang et al., 2006; Huang e al., 2020) after being collected on a quartz filter, using the same thermal/ or thermal-optical instrumentation as EC in the IMPROVE, NAPS, EMEP and CABM measurement networks. These OC measurements have approximately $20 \%$ uncertainty (Sharma et al, 2017). 
Table S1. Arctic monitoring stations and the observed aerosol species used in model evaluation

\begin{tabular}{|l|c|c|l|}
\hline Stations & Latitude & Longitude & Aerosol Species \\
\hline Alert (B1, O1, S1) & 82.47 & -62.5 & $\mathrm{BC}, \mathrm{OC}, \mathrm{SO}^{2-}$ \\
\hline Denali (B2, O2, S2) & 63.11 & -151.19 & $\mathrm{BC}, \mathrm{OC}, \mathrm{SO}^{2-}$ \\
\hline Fairbanks (B3, S3) & 64.84 & -147.72 & $\mathrm{BC}, \mathrm{SO} 4^{2-}$ \\
\hline Gates of the Arctic (B4, O3, S4) & 67.91 & -153.46 & $\mathrm{BC}, \mathrm{OC}, \mathrm{SO}^{2-}$ \\
\hline Gruvebadet (B5) & 79.00 & 12.00 & $\mathrm{BC}$ \\
\hline Hurdal (B6, O4, S5) & 60.44 & 11.07 & $\mathrm{BC}, \mathrm{OC}^{2-} \mathrm{SO} 4^{2-}$ \\
\hline Karasjok (S6) & 69.47 & 25.51 & $\mathrm{SO} 4{ }^{2-}$ \\
\hline Karvatn (B7, O5, S7) & 62.78 & 8.88 & $\mathrm{BC}, \mathrm{OC}^{2}, \mathrm{SO}^{2-}$ \\
\hline TrapperCreek (B8, O6, S8) & 62.32 & -150.23 & $\mathrm{BC}, \mathrm{OC}^{2}, \mathrm{SO}^{2-}$ \\
\hline Utqiagvik (B9, S10) & 71.29 & -156.79 & $\mathrm{BC}, \mathrm{SO} 4^{2-}$ \\
\hline Villum Research Station (B10, S11) & 81.60 & -16.67 & $\mathrm{EC}, \mathrm{SO} 4^{2-}$ \\
\hline ZeppelinMountain (B11, S12) & 78.91 & 11.89 & $\mathrm{BC}, \mathrm{SO} 4^{2-}$ \\
\hline
\end{tabular}


Table S2. Arctic Aeronet stations used in model evaluation

\begin{tabular}{|c|c|c|}
\hline Stations & Latitude & Longitude \\
\hline Andenes (A1) & 69.28 & 16.01 \\
\hline ARM_Oliktok_AK (A2) & 70.50 & 210.12 \\
\hline Barrow (A3) & 71.31 & 203.34 \\
\hline Bonanza Creek (A4) & 64.74 & 211.68 \\
\hline Helsinki (A5) & 60.20 & 24.96 \\
\hline Hornsund (A6) & 77.00 & 15.54 \\
\hline Hyytiala (A7) & 61.85 & 24.30 \\
\hline Iqaluit (A8) & 63.75 & 291.46 \\
\hline Ittoqqortoormiit (A9) & 70.48 & 338.05 \\
\hline Kangerlussuaq (A10) & 67.00 & 309.38 \\
\hline Kuopio (A11) & 62.89 & 27.63 \\
\hline Narsarsuaq (A12) & 61.16 & 314.58 \\
\hline Opal (A13) & 79.99 & 274.06 \\
\hline Pearl (A14) & 80.05 & 273.58 \\
\hline Resolute_Bay (A15) & 74.71 & 265.03 \\
\hline Sodankyla (A16) & 67.37 & 26.63 \\
\hline Thule (A17) & 76.52 & 291.23 \\
\hline Tiksi (A18) & 71.59 & 128.92 \\
\hline Yakutsk (A19) & 61.66 & 129.37 \\
\hline Yellowknife_Aurora (A20) & 62.45 & 245.62 \\
\hline
\end{tabular}


Table S3. Model evaluation over the individual monitoring stations for black carbon (BC). Statistics used are mean bias $(M B)$, mean gross error $(M G E)$, normalized mean bias $(N M B)$, normalized mean gross error (NMGE), root mean square error (RMSE), and Pearson's correlation $(r)$.

\begin{tabular}{|l|c|c|c|c|c|c|l|}
\hline Station & $M B$ & $M G E$ & $N M B$ & $N M G E$ & $R M S E$ & $r$ & Simulation \\
\hline Alert & -0.04 & 0.04 & -0.95 & 0.96 & 0.05 & 0.01 & Eclipse_AMIP \\
\hline Alert & -0.04 & 0.04 & -0.96 & 0.96 & 0.05 & 0.43 & Eclipse_AMIP_NCEP \\
\hline Alert & -0.04 & 0.04 & -0.95 & 0.95 & 0.05 & 0.13 & Eclipse_CplHist1 \\
\hline Alert & -0.04 & 0.04 & -0.95 & 0.95 & 0.05 & 0.37 & Eclipse_Cp1Hist2 \\
\hline Alert & -0.04 & 0.04 & -0.95 & 0.95 & 0.05 & 0.30 & Eclipse_CplHist3 \\
\hline Alert & -0.04 & 0.04 & -0.94 & 0.94 & 0.05 & 0.09 & CMIP6_Cp1_Hist \\
\hline Denali & -0.06 & 0.06 & -0.73 & 0.74 & 0.11 & 0.70 & Eclipse_AMIP \\
\hline Denali & -0.05 & 0.05 & -0.62 & 0.66 & 0.09 & 0.77 & Eclipse_AMIP_NCEP \\
\hline Denali & -0.05 & 0.06 & -0.70 & 0.72 & 0.11 & 0.60 & Eclipse_CplHist1 \\
\hline Denali & -0.05 & 0.05 & -0.68 & 0.70 & 0.11 & 0.60 & Eclipse_Cp1Hist2 \\
\hline Denali & -0.05 & 0.05 & -0.68 & 0.70 & 0.10 & 0.71 & Eclipse_CplHist3 \\
\hline Denali & -0.05 & 0.05 & -0.65 & 0.69 & 0.10 & 0.65 & CMIP6_Cp1_Hist \\
\hline Fairbanks & -0.02 & 0.14 & -0.19 & 1.28 & 0.33 & -0.09 & Eclipse_AMIP \\
\hline Fairbanks & 0.03 & 0.16 & 0.31 & 1.50 & 0.49 & -0.08 & Eclipse_AMIP_NCEP \\
\hline Fairbanks & 0.00 & 0.16 & 0.04 & 1.50 & 0.46 & -0.09 & Eclipse_CplHist1 \\
\hline Fairbanks & -0.02 & 0.14 & -0.16 & 1.31 & 0.32 & -0.10 & Eclipse_CplHist2 \\
\hline Fairbanks & -0.01 & 0.15 & -0.06 & 1.39 & 0.39 & -0.09 & Eclipse_Cp1Hist3 \\
\hline Fairbanks & 0.00 & 0.16 & -0.04 & 1.44 & 0.40 & -0.10 & CMIP6_Cp1_Hist \\
\hline GatesoftheArctic & -0.05 & 0.05 & -0.74 & 0.77 & 0.13 & 0.84 & Eclipse_AMIP \\
\hline
\end{tabular}




\begin{tabular}{|l|c|c|c|c|c|c|l|}
\hline GatesoftheArctic & -0.05 & 0.05 & -0.68 & 0.69 & 0.12 & 0.74 & Eclipse_AMIP_NCEP \\
\hline GatesoftheArctic & -0.04 & 0.04 & -0.66 & 0.66 & 0.12 & 0.80 & Eclipse_CplHist1 \\
\hline GatesoftheArctic & -0.04 & 0.04 & -0.64 & 0.65 & 0.11 & 0.84 & Eclipse_CplHist2 \\
\hline GatesoftheArctic & -0.04 & 0.04 & -0.64 & 0.66 & 0.12 & 0.76 & Eclipse_CplHist3 \\
\hline GatesoftheArctic & -0.04 & 0.04 & -0.59 & 0.61 & 0.14 & 0.53 & CMIP6_Cp1_Hist \\
\hline Gruvebadet & -0.04 & 0.04 & -0.93 & 0.93 & 0.06 & 0.43 & Eclipse_AMIP \\
\hline Gruvebadet & -0.05 & 0.05 & -0.94 & 0.94 & 0.06 & 0.42 & Eclipse_AMIP_NCEP \\
\hline Gruvebadet & -0.04 & 0.04 & -0.93 & 0.93 & 0.06 & 0.34 & Eclipse_CplHist1 \\
\hline Gruvebadet & -0.04 & 0.04 & -0.91 & 0.91 & 0.05 & 0.23 & Eclipse_CplHist2 \\
\hline Gruvebadet & -0.05 & 0.05 & -0.94 & 0.94 & 0.06 & 0.43 & Eclipse_CplHist3 \\
\hline Gruvebadet & -0.04 & 0.04 & -0.87 & 0.87 & 0.05 & 0.25 & CMIP6_Cpl_Hist \\
\hline Hurdal & -0.06 & 0.06 & -0.43 & 0.44 & 0.07 & 0.64 & Eclipse_AMIP \\
\hline Hurdal & -0.06 & 0.06 & -0.45 & 0.46 & 0.08 & 0.63 & Eclipse_AMIP_NCEP \\
\hline Hurdal & -0.06 & 0.06 & -0.45 & 0.45 & 0.08 & 0.62 & Eclipse_CplHist1 \\
\hline Hurdal & -0.06 & 0.06 & -0.42 & 0.46 & 0.08 & 0.54 & Eclipse_CplHist2 \\
\hline Hurdal & -0.06 & 0.06 & -0.45 & 0.45 & 0.08 & 0.66 & Eclipse_CplHist3 \\
\hline Hurdal & -0.03 & 0.04 & -0.21 & 0.28 & 0.05 & 0.61 & CMIP6_Cp1_Hist \\
\hline Karvatn & -0.02 & 0.03 & -0.35 & 0.52 & 0.04 & -0.09 & Eclipse_AMIP \\
\hline Karvatn & -0.02 & 0.02 & -0.29 & 0.41 & 0.03 & 0.36 & Eclipse_AMIP_NCEP \\
\hline Karvatn & -0.02 & 0.03 & -0.36 & 0.47 & 0.03 & -0.05 & Eclipse_CplHist1 \\
\hline Karvatn & -0.02 & 0.03 & -0.28 & 0.50 & 0.03 & -0.13 & Eclipse_CplHist2 \\
\hline Karvatn & 0.03 & -0.37 & 0.47 & 0.03 & 0.08 & Eclipse_CplHist3 \\
\hline Karvatn & -0.31 & 0.43 & 0.03 & 0.04 & CMIP6_Cpl_Hist \\
\hline
\end{tabular}




\begin{tabular}{|l|c|c|c|c|c|c|l|}
\hline TrapperCreek & -0.03 & 0.04 & -0.61 & 0.69 & 0.05 & 0.69 & Eclipse_AMIP \\
\hline TrapperCreek & -0.02 & 0.04 & -0.42 & 0.66 & 0.05 & 0.72 & Eclipse_AMIP_NCEP \\
\hline TrapperCreek & -0.03 & 0.04 & -0.57 & 0.70 & 0.05 & 0.57 & Eclipse_CplHist1 \\
\hline TrapperCreek & -0.03 & 0.04 & -0.54 & 0.70 & 0.06 & 0.47 & Eclipse_Cp1Hist2 \\
\hline TrapperCreek & -0.03 & 0.04 & -0.52 & 0.69 & 0.05 & 0.62 & Eclipse_CplHist3 \\
\hline TrapperCreek & -0.03 & 0.04 & -0.46 & 0.76 & 0.07 & 0.53 & CMIP6_Cp1_Hist \\
\hline Utqiagvik & -0.02 & 0.03 & -0.74 & 0.90 & 0.04 & -0.23 & Eclipse_AMIP \\
\hline Utqiagvik & -0.02 & 0.03 & -0.67 & 0.88 & 0.04 & -0.12 & Eclipse_AMIP_NCEP \\
\hline Utqiagvik & -0.02 & 0.03 & -0.67 & 0.94 & 0.04 & -0.23 & Eclipse_CplHist1 \\
\hline Utqiagvik & -0.02 & 0.03 & -0.69 & 0.92 & 0.04 & -0.21 & Eclipse_Cp1Hist2 \\
\hline Utqiagvik & -0.02 & 0.03 & -0.69 & 0.93 & 0.04 & -0.21 & Eclipse_CplHist3 \\
\hline Utqiagvik & 0.00 & 0.03 & 0.10 & 0.83 & 0.04 & -0.04 & CMIP6_Cp1_Hist \\
\hline ZeppelinMountain & -0.02 & 0.02 & -0.83 & 0.83 & 0.03 & 0.38 & Eclipse_AMIP \\
\hline ZeppelinMountain & -0.02 & 0.02 & -0.84 & 0.84 & 0.03 & 0.48 & Eclipse_AMIP_NCEP \\
\hline ZeppelinMountain & -0.02 & 0.02 & -0.80 & 0.81 & 0.03 & 0.32 & Eclipse_CplHist1 \\
\hline ZeppelinMountain & -0.02 & 0.02 & -0.81 & 0.82 & 0.03 & 0.40 & Eclipse_CplHist2 \\
\hline ZeppelinMountain & -0.02 & 0.02 & -0.81 & 0.82 & 0.03 & 0.39 & Eclipse_CplHist3 \\
\hline ZeppelinMountain & -0.02 & 0.02 & -0.74 & 0.77 & 0.03 & 0.23 & CMIP6_Cp1_Hist \\
\hline
\end{tabular}


Table S4. Model evaluation over the individual monitoring stations for organic carbon (OC). Statistics used are mean bias $(M B)$, mean gross error $(M G E)$, normalized mean bias $(N M B)$, normalized mean gross error ( $N M G E)$, root mean square error (RMSE), and Pearson's correlation $(r)$.

\begin{tabular}{|l|c|c|c|c|c|c|l|}
\hline Station & $M B$ & $M G E$ & $N M B$ & $N M G E$ & $R M S E$ & $r$ & Simulation \\
\hline Alert & -0.13 & 0.14 & -0.83 & 0.88 & 0.16 & 0.01 & Eclipse_AMIP \\
\hline Alert & -0.13 & 0.13 & -0.83 & 0.84 & 0.15 & 0.24 & Eclipse_AMIP_NCEP \\
\hline Alert & -0.12 & 0.13 & -0.79 & 0.81 & 0.15 & 0.04 & Eclipse_Cp1Hist1 \\
\hline Alert & -0.12 & 0.13 & -0.79 & 0.82 & 0.15 & 0.11 & Eclipse_Cp1Hist2 \\
\hline Alert & -0.12 & 0.12 & -0.79 & 0.81 & 0.15 & 0.21 & Eclipse_Cp1Hist3 \\
\hline Alert & -0.11 & 0.12 & -0.72 & 0.77 & 0.14 & 0.09 & CMIP6_Cp1_Hist \\
\hline Denali & -0.20 & 0.44 & -0.31 & 0.66 & 1.59 & 0.69 & Eclipse_AMIP \\
\hline Denali & 0.03 & 0.46 & 0.05 & 0.69 & 1.34 & 0.77 & Eclipse_AMIP_NCEP \\
\hline Denali & -0.10 & 0.49 & -0.15 & 0.74 & 1.68 & 0.59 & Eclipse_Cp1Hist1 \\
\hline Denali & -0.07 & 0.48 & -0.11 & 0.73 & 1.76 & 0.52 & Eclipse_CplHist2 \\
\hline Denali & -0.05 & 0.45 & -0.07 & 0.68 & 1.55 & 0.67 & Eclipse_CplHist3 \\
\hline Denali & 0.17 & 0.56 & 0.26 & 0.84 & 1.74 & 0.62 & CMIP6_Cp1_Hist \\
\hline GatesOfTheArctic & -0.28 & 0.38 & -0.44 & 0.59 & 0.68 & 0.90 & Eclipse_AMIP \\
\hline GatesOfTheArctic & -0.18 & 0.30 & -0.29 & 0.47 & 0.55 & 0.93 & Eclipse_AMIP_NCEP \\
\hline GatesOfTheArctic & -0.16 & 0.30 & -0.25 & 0.46 & 0.52 & 0.94 & Eclipse_CplHist1 \\
\hline GatesOfTheArctic & -0.13 & 0.23 & -0.20 & 0.37 & 0.41 & 0.96 & Eclipse_CplHist2 \\
\hline GatesOfTheArctic & -0.12 & 0.26 & -0.19 & 0.41 & 0.43 & 0.95 & Eclipse_CplHist3 \\
\hline GatesOfTheArctic & -0.06 & 0.38 & -0.10 & 0.59 & 1.01 & 0.84 & CMIP6_Cp1_Hist \\
\hline
\end{tabular}




\begin{tabular}{|l|c|c|c|c|c|c|l|}
\hline Hurdal & -0.60 & 0.73 & -0.52 & 0.64 & 0.98 & -0.50 & Eclipse_AMIP \\
\hline Hurdal & -0.65 & 0.77 & -0.57 & 0.67 & 0.98 & -0.26 & Eclipse_AMIP_NCEP \\
\hline Hurdal & -0.59 & 0.69 & -0.51 & 0.61 & 0.93 & -0.54 & Eclipse_Cp1Hist1 \\
\hline Hurdal & -0.58 & 0.72 & -0.50 & 0.63 & 0.92 & -0.48 & Eclipse_CplHist2 \\
\hline Hurdal & -0.66 & 0.74 & -0.57 & 0.65 & 0.97 & -0.49 & Eclipse_CplHist3 \\
\hline Hurdal & -0.44 & 0.65 & -0.39 & 0.57 & 0.87 & -0.62 & CMIP6_Cp1_Hist \\
\hline Karvatn & -0.67 & 0.67 & -0.82 & 0.83 & 0.89 & -0.24 & Eclipse_AMIP \\
\hline Karvatn & -0.64 & 0.66 & -0.79 & 0.80 & 0.88 & -0.25 & Eclipse_AMIP_NCEP \\
\hline Karvatn & -0.68 & 0.68 & -0.83 & 0.83 & 0.89 & -0.35 & Eclipse_Cp1Hist1 \\
\hline Karvatn & -0.66 & 0.67 & -0.81 & 0.82 & 0.88 & -0.23 & Eclipse_Cp1Hist2 \\
\hline Karvatn & -0.68 & 0.69 & -0.84 & 0.84 & 0.89 & -0.20 & Eclipse_CplHist3 \\
\hline Karvatn & -0.62 & 0.63 & -0.76 & 0.77 & 0.85 & -0.36 & CMIP6_Cp1_Hist \\
\hline TrapperCreek & 0.06 & 0.32 & 0.13 & 0.73 & 0.71 & 0.74 & Eclipse_AMIP \\
\hline TrapperCreek & 0.33 & 0.49 & 0.76 & 1.12 & 1.17 & 0.80 & Eclipse_AMIP_NCEP \\
\hline TrapperCreek & 0.14 & 0.39 & 0.32 & 0.88 & 0.86 & 0.63 & Eclipse_Cp1Hist1 \\
\hline TrapperCreek & 0.20 & 0.44 & 0.45 & 1.00 & 1.05 & 0.52 & Eclipse_Cp1Hist2 \\
\hline TrapperCreek & 0.23 & 0.41 & 0.53 & 0.92 & 0.99 & 0.68 & Eclipse_Cp1Hist3 \\
\hline TrapperCreek & 0.50 & 0.61 & 1.13 & 1.39 & 1.97 & 0.63 & CMIP6_Cp1_Hist \\
\hline
\end{tabular}


Table S5. Model evaluation over the individual monitoring stations for sulfate (SO4$\left.{ }^{2-}\right)$. Statistics used are mean bias $(M B)$, mean gross error $(M G E)$, normalized mean bias $(N M B)$, normalized mean gross error (NMGE), root mean square error ( $R M S E)$, and Pearson's correlation $(r)$.

\begin{tabular}{|l|c|c|c|c|c|c|l|}
\hline Station & $M B$ & $M G E$ & $N M B$ & $N M G E$ & $R M S E$ & $r$ & Simulation \\
\hline Alert & -0.34 & 0.37 & -0.72 & 0.77 & 0.48 & 0.38 & Eclipse_AMIP \\
\hline Alert & -0.37 & 0.37 & -0.77 & 0.78 & 0.49 & 0.50 & Eclipse_AMIP_NCEP \\
\hline Alert & -0.34 & 0.36 & -0.72 & 0.76 & 0.48 & 0.44 & Eclipse_CplHist1 \\
\hline Alert & -0.34 & 0.35 & -0.72 & 0.75 & 0.47 & 0.48 & Eclipse_CplHist2 \\
\hline Alert & -0.34 & 0.36 & -0.72 & 0.75 & 0.47 & 0.46 & Eclipse_CplHist3 \\
\hline Alert & -0.32 & 0.34 & -0.68 & 0.72 & 0.45 & 0.51 & CMIP6_Cp1_Hist \\
\hline Denali & -0.15 & 0.17 & -0.46 & 0.53 & 0.24 & 0.38 & Eclipse_AMIP \\
\hline Denali & -0.16 & 0.17 & -0.50 & 0.52 & 0.23 & 0.67 & Eclipse_AMIP_NCEP \\
\hline Denali & -0.14 & 0.16 & -0.45 & 0.50 & 0.23 & 0.48 & Eclipse_CplHist1 \\
\hline Denali & -0.14 & 0.16 & -0.43 & 0.49 & 0.23 & 0.46 & Eclipse_CplHist2 \\
\hline Denali & -0.14 & 0.16 & -0.42 & 0.48 & 0.22 & 0.51 & Eclipse_CplHist3 \\
\hline Denali & -0.09 & 0.14 & -0.28 & 0.43 & 0.20 & 0.54 & CMIP6_Cp1_Hist \\
\hline Fairbanks & 0.00 & 0.19 & 0.02 & 1.04 & 0.24 & -0.36 & Eclipse_AMIP \\
\hline Fairbanks & 0.02 & 0.20 & 0.09 & 1.07 & 0.25 & -0.28 & Eclipse_AMIP_NCEP \\
\hline Fairbanks & 0.00 & 0.19 & 0.03 & 1.05 & 0.24 & -0.35 & Eclipse_CplHist1 \\
\hline Fairbanks & 0.01 & 0.19 & 0.06 & 1.02 & 0.23 & -0.34 & Eclipse_CplHist2 \\
\hline Fairbanks & 0.01 & 0.19 & 0.06 & 1.02 & 0.23 & -0.31 & Eclipse_CplHist3 \\
\hline Fairbanks & 0.08 & 0.23 & 0.46 & 1.22 & 0.27 & -0.32 & CMIP6_Cp1_Hist \\
\hline GatesoftheArctic & -0.25 & 0.26 & -0.64 & 0.66 & 0.36 & 0.53 & Eclipse_AMIP \\
\hline
\end{tabular}




\begin{tabular}{|l|c|c|c|c|c|c|l|}
\hline GatesoftheArctic & -0.26 & 0.26 & -0.65 & 0.66 & 0.36 & 0.64 & Eclipse_AMIP_NCEP \\
\hline GatesoftheArctic & -0.25 & 0.26 & -0.64 & 0.66 & 0.37 & 0.49 & Eclipse_CplHist1 \\
\hline GatesoftheArctic & -0.24 & 0.25 & -0.61 & 0.63 & 0.36 & 0.48 & Eclipse_CplHist2 \\
\hline GatesoftheArctic & -0.25 & 0.25 & -0.63 & 0.64 & 0.36 & 0.52 & Eclipse_CplHist3 \\
\hline GatesoftheArctic & -0.20 & 0.22 & -0.51 & 0.55 & 0.31 & 0.64 & CMIP6_Cp1_Hist \\
\hline Hurdal & -0.55 & 0.57 & -0.60 & 0.62 & 0.72 & 0.28 & Eclipse_AMIP \\
\hline Hurdal & -0.54 & 0.55 & -0.59 & 0.59 & 0.65 & 0.69 & Eclipse_AMIP_NCEP \\
\hline Hurdal & -0.55 & 0.58 & -0.60 & 0.63 & 0.72 & 0.29 & Eclipse_CplHist1 \\
\hline Hurdal & -0.52 & 0.55 & -0.57 & 0.60 & 0.69 & 0.34 & Eclipse_CplHist2 \\
\hline Hurdal & -0.54 & 0.57 & -0.59 & 0.62 & 0.72 & 0.27 & Eclipse_CplHist3 \\
\hline Hurdal & -0.49 & 0.53 & -0.53 & 0.57 & 0.68 & 0.29 & CMIP6_Cp1_Hist \\
\hline Karasjok & -0.48 & 0.52 & -0.58 & 0.61 & 0.67 & 0.24 & Eclipse_AMIP \\
\hline Karasjok & -0.50 & 0.50 & -0.60 & 0.60 & 0.63 & 0.55 & Eclipse_AMIP_NCEP \\
\hline Karasjok & -0.46 & 0.49 & -0.55 & 0.58 & 0.65 & 0.28 & Eclipse_CplHist1 \\
\hline Karasjok & -0.47 & 0.48 & -0.56 & 0.57 & 0.65 & 0.29 & Eclipse_CplHist2 \\
\hline Karasjok & -0.46 & 0.48 & -0.55 & 0.57 & 0.64 & 0.34 & Eclipse_CplHist3 \\
\hline Karasjok & -0.38 & 0.42 & -0.46 & 0.50 & 0.59 & 0.35 & CMIP6_Cp1_Hist \\
\hline Karvatn & -0.16 & 0.26 & -0.29 & 0.49 & 0.39 & 0.39 & Eclipse_AMIP \\
\hline Karvatn & -0.19 & 0.20 & -0.35 & 0.38 & 0.30 & 0.76 & Eclipse_AMIP_NCEP \\
\hline Karvatn & -0.19 & 0.25 & -0.35 & 0.47 & 0.38 & 0.44 & Eclipse_CplHist1 \\
\hline Karvatn & -0.16 & 0.24 & -0.31 & 0.46 & 0.37 & 0.45 & Eclipse_CplHist2 \\
\hline Karvatn & 0.24 & -0.34 & 0.45 & 0.36 & 0.50 & Eclipse_CplHist3 \\
\hline Karvatn & -0.24 & 0.42 & 0.35 & 0.48 & CMIP6_Cp1_Hist \\
\hline
\end{tabular}




\begin{tabular}{|l|c|c|c|c|c|c|l|}
\hline TrapperCreek & -0.16 & 0.17 & -0.49 & 0.52 & 0.25 & 0.52 & Eclipse_AMIP \\
\hline TrapperCreek & -0.17 & 0.17 & -0.52 & 0.52 & 0.23 & 0.75 & Eclipse_AMIP_NCEP \\
\hline TrapperCreek & -0.16 & 0.17 & -0.49 & 0.50 & 0.24 & 0.61 & Eclipse_CplHist1 \\
\hline TrapperCreek & -0.15 & 0.17 & -0.46 & 0.51 & 0.24 & 0.51 & Eclipse_CplHist2 \\
\hline TrapperCreek & -0.15 & 0.16 & -0.46 & 0.49 & 0.23 & 0.60 & Eclipse_CplHist3 \\
\hline TrapperCreek & -0.11 & 0.14 & -0.32 & 0.42 & 0.21 & 0.60 & CMIP6_Cp1_Hist \\
\hline Tustervatn & -0.22 & 0.29 & -0.39 & 0.52 & 0.40 & 0.37 & Eclipse_AMIP \\
\hline Tustervatn & -0.26 & 0.27 & -0.46 & 0.47 & 0.35 & 0.72 & Eclipse_AMIP_NCEP \\
\hline Tustervatn & -0.25 & 0.29 & -0.44 & 0.52 & 0.42 & 0.36 & Eclipse_Cp1Hist1 \\
\hline Tustervatn & -0.23 & 0.29 & -0.40 & 0.51 & 0.40 & 0.38 & Eclipse_CplHist2 \\
\hline Tustervatn & -0.24 & 0.28 & -0.43 & 0.50 & 0.40 & 0.41 & Eclipse_CplHist3 \\
\hline Tustervatn & -0.18 & 0.26 & -0.32 & 0.46 & 0.38 & 0.40 & CMIP6_Cp1_Hist \\
\hline Utqiagvik & -0.25 & 0.29 & -0.61 & 0.70 & 0.37 & 0.16 & Eclipse_AMIP \\
\hline Utqiagvik & -0.23 & 0.28 & -0.56 & 0.68 & 0.35 & 0.24 & Eclipse_AMIP_NCEP \\
\hline Utqiagvik & -0.24 & 0.29 & -0.59 & 0.69 & 0.37 & 0.13 & Eclipse_Cp1Hist1 \\
\hline Utqiagvik & -0.24 & 0.29 & -0.59 & 0.69 & 0.37 & 0.15 & Eclipse_CplHist2 \\
\hline Utqiagvik & -0.25 & 0.28 & -0.60 & 0.68 & 0.36 & 0.22 & Eclipse_CplHist3 \\
\hline Utqiagvik & -0.20 & 0.26 & -0.48 & 0.63 & 0.33 & 0.27 & CMIP6_Cp1_Hist \\
\hline VillumNord & -0.26 & 0.30 & -0.64 & 0.72 & 0.41 & 0.41 & Eclipse_AMIP \\
\hline VillumNord & -0.28 & 0.30 & -0.68 & 0.72 & 0.41 & 0.49 & Eclipse_AMIP_NCEP \\
\hline VillumNord & -0.26 & 0.29 & -0.63 & 0.72 & 0.41 & 0.41 & Eclipse_CplHist1 \\
\hline VillumNord & 0.29 & -0.64 & 0.70 & 0.40 & 0.48 & Eclipse_Cp1Hist2 \\
\hline VillumNord & -0.64 & 0.70 & 0.40 & 0.50 & Eclipse_CplHist3 \\
\hline
\end{tabular}




\begin{tabular}{|l|c|c|c|c|c|c|l|}
\hline VillumNord & -0.24 & 0.27 & -0.59 & 0.67 & 0.38 & 0.51 & CMIP6_Cp1_Hist \\
\hline ZeppelinMountain & 0.04 & 0.11 & 0.29 & 0.80 & 0.16 & 0.34 & Eclipse_AMIP \\
\hline ZeppelinMountain & 0.03 & 0.08 & 0.18 & 0.62 & 0.11 & 0.59 & Eclipse_AMIP_NCEP \\
\hline ZeppelinMountain & 0.05 & 0.11 & 0.39 & 0.80 & 0.15 & 0.49 & Eclipse_CplHist1 \\
\hline ZeppelinMountain & 0.05 & 0.11 & 0.37 & 0.79 & 0.15 & 0.47 & Eclipse_CplHist2 \\
\hline ZeppelinMountain & 0.05 & 0.10 & 0.33 & 0.74 & 0.15 & 0.52 & Eclipse_CplHist3 \\
\hline ZeppelinMountain & 0.08 & 0.12 & 0.56 & 0.89 & 0.17 & 0.52 & CMIP6_Cp1_Hist \\
\hline
\end{tabular}


Table S6. Model evaluation over the individual Aeronet monitoring stations for aerosol optical depth at $550 \mathrm{~nm}$. Statistics used are mean bias $(M B)$, mean gross error $(M G E)$, normalized mean bias $(N M B)$, normalized mean gross error $(N M G E)$, root mean square error (RMSE), and Pearson's correlation $(r)$.

\begin{tabular}{|c|c|c|c|c|c|c|c|}
\hline Station & $M B$ & $M G E$ & $N M B$ & $N M G E$ & $R M S E$ & $r$ & Simulation \\
\hline Andenes & -0.02 & 0.05 & -0.22 & 0.49 & 0.06 & 0.03 & Eclipse_AMIP \\
\hline Andenes & -0.03 & 0.04 & -0.30 & 0.36 & 0.04 & 0.35 & Eclipse_AMIP_NCEP \\
\hline Andenes & -0.04 & 0.05 & -0.43 & 0.46 & 0.05 & 0.04 & Eclipse_CplHist1 \\
\hline Andenes & -0.03 & 0.04 & -0.32 & 0.43 & 0.05 & 0.22 & Eclipse_CplHist2 \\
\hline Andenes & -0.01 & 0.05 & -0.13 & 0.47 & 0.06 & -0.09 & Eclipse_CplHist3 \\
\hline Andenes & -0.03 & 0.04 & -0.29 & 0.36 & 0.04 & 0.20 & CMIP6_Cpl_Hist \\
\hline ARM_HyytialaFinland & 0.00 & 0.06 & 0.00 & 0.65 & 0.07 & -0.73 & Eclipse_AMIP \\
\hline ARM_HyytialaFinland & -0.02 & 0.04 & -0.23 & 0.46 & 0.04 & -0.51 & Eclipse_AMIP_NCEP \\
\hline ARM_HyytialaFinland & -0.02 & 0.05 & -0.21 & 0.55 & 0.05 & -0.68 & Eclipse_CplHist1 \\
\hline ARM_HyytialaFinland & -0.02 & 0.05 & -0.18 & 0.62 & 0.05 & -0.88 & Eclipse_CplHist2 \\
\hline ARM_HyytialaFinland & -0.03 & 0.04 & -0.30 & 0.42 & 0.04 & -0.46 & Eclipse_CplHist3 \\
\hline ARM_HyytialaFinland & -0.02 & 0.04 & -0.20 & 0.44 & 0.04 & -0.73 & CMIP6_Cpl_Hist \\
\hline Barrow & -0.08 & 0.08 & -0.55 & 0.58 & 0.10 & 0.34 & Eclipse_AMIP \\
\hline Barrow & -0.09 & 0.09 & -0.62 & 0.63 & 0.11 & 0.44 & Eclipse_AMIP_NCEP \\
\hline Barrow & -0.09 & 0.09 & -0.65 & 0.66 & 0.12 & 0.22 & Eclipse_CplHist1 \\
\hline Barrow & -0.09 & 0.09 & -0.64 & 0.66 & 0.11 & 0.35 & Eclipse_CplHist2 \\
\hline Barrow & -0.08 & 0.09 & -0.62 & 0.63 & 0.11 & 0.28 & Eclipse_CplHist3 \\
\hline Barrow & -0.08 & 0.08 & -0.56 & 0.59 & 0.11 & 0.02 & CMIP6_Cpl_Hist \\
\hline
\end{tabular}




\begin{tabular}{|l|c|c|c|c|c|l|l|}
\hline Bonanza_Creek & -0.08 & 0.09 & -0.57 & 0.61 & 0.16 & 0.13 & Eclipse_AMIP \\
\hline Bonanza_Creek & -0.08 & 0.08 & -0.56 & 0.57 & 0.15 & 0.52 & Eclipse_AMIP_NCEP \\
\hline Bonanza_Creek & -0.10 & 0.10 & -0.67 & 0.70 & 0.17 & 0.00 & Eclipse_CplHist1 \\
\hline Bonanza_Creek & -0.10 & 0.10 & -0.66 & 0.66 & 0.17 & 0.28 & Eclipse_CplHist2 \\
\hline Bonanza_Creek & -0.09 & 0.09 & -0.64 & 0.66 & 0.17 & 0.31 & Eclipse_CplHist3 \\
\hline Bonanza_Creek & -0.09 & 0.09 & -0.59 & 0.62 & 0.17 & 0.09 & CMIP6_Cpl_Hist \\
\hline Helsinki & 0.00 & 0.06 & -0.05 & 0.64 & 0.08 & -0.51 & Eclipse_AMIP \\
\hline Helsinki & -0.02 & 0.04 & -0.25 & 0.47 & 0.05 & -0.35 & Eclipse_AMIP_NCEP \\
\hline Helsinki & 0.00 & 0.07 & 0.02 & 0.75 & 0.10 & -0.54 & Eclipse_CplHist1 \\
\hline Helsinki & -0.03 & 0.04 & -0.27 & 0.39 & 0.04 & -0.14 & Eclipse_CplHist2 \\
\hline Helsinki & -0.02 & 0.05 & -0.19 & 0.50 & 0.05 & -0.68 & Eclipse_CplHist3 \\
\hline Helsinki & -0.01 & 0.06 & -0.10 & 0.59 & 0.07 & -0.66 & CMIP6_Cpl_Hist \\
\hline Hornsund & -0.04 & 0.04 & -0.38 & 0.42 & 0.05 & 0.40 & Eclipse_AMIP \\
\hline Hornsund & -0.05 & 0.05 & -0.48 & 0.48 & 0.06 & 0.31 & Eclipse_AMIP_NCEP \\
\hline Hornsund & -0.04 & 0.05 & -0.43 & 0.49 & 0.06 & 0.07 & Eclipse_CplHist1 \\
\hline Hornsund & -0.04 & 0.05 & -0.39 & 0.48 & 0.06 & 0.14 & Eclipse_CplHist2 \\
\hline Hornsund & -0.03 & 0.04 & -0.31 & 0.41 & 0.06 & 0.25 & Eclipse_CplHist3 \\
\hline Hornsund & -0.04 & 0.05 & -0.43 & 0.48 & 0.06 & -0.07 & CMIP6_Cpl_Hist \\
\hline Hyytiala & -0.02 & 0.04 & -0.23 & 0.44 & 0.05 & -0.31 & Eclipse_AMIP \\
\hline Hyytiala & -0.03 & 0.05 & -0.26 & 0.50 & 0.06 & -0.50 & Eclipse_AMIP_NCEP \\
\hline Hyytiala & 0.00 & 0.06 & -0.03 & 0.61 & 0.10 & -0.32 & Eclipse_CplHist1 \\
\hline Hyytiala & -0.22 & 0.35 & 0.04 & 0.09 & Eclipse_CplHist2 \\
\hline Hyytiala & 0.32 & 0.44 & 0.05 & -0.09 & Eclipse_CplHist3 \\
\hline
\end{tabular}




\begin{tabular}{|l|c|c|c|c|c|c|l|}
\hline Hyytiala & -0.02 & 0.05 & -0.18 & 0.49 & 0.06 & -0.31 & CMIP6_Cpl_Hist \\
\hline Iqaluit & -0.01 & 0.04 & -0.10 & 0.42 & 0.06 & 0.01 & Eclipse_AMIP \\
\hline Iqaluit & -0.02 & 0.05 & -0.20 & 0.49 & 0.07 & 0.07 & Eclipse_AMIP_NCEP \\
\hline Iqaluit & -0.03 & 0.04 & -0.35 & 0.45 & 0.06 & -0.13 & Eclipse_CplHist1 \\
\hline Iqaluit & -0.02 & 0.04 & -0.18 & 0.47 & 0.06 & 0.18 & Eclipse_CplHist2 \\
\hline Iqaluit & -0.02 & 0.05 & -0.21 & 0.52 & 0.07 & -0.17 & Eclipse_CplHist3 \\
\hline Iqaluit & -0.02 & 0.04 & -0.22 & 0.39 & 0.06 & 0.14 & CMIP6_Cpl_Hist \\
\hline Ittoqqortoormiit & -0.01 & 0.02 & -0.20 & 0.24 & 0.02 & 0.71 & Eclipse_AMIP \\
\hline Ittoqqortoormiit & -0.03 & 0.03 & -0.35 & 0.35 & 0.03 & 0.72 & Eclipse_AMIP_NCEP \\
\hline Ittoqqortoormiit & -0.03 & 0.04 & -0.43 & 0.49 & 0.04 & -0.07 & Eclipse_CplHist1 \\
\hline Ittoqqortoormiit & -0.03 & 0.03 & -0.41 & 0.44 & 0.03 & 0.02 & Eclipse_CplHist2 \\
\hline Ittoqqortoormiit & -0.02 & 0.03 & -0.34 & 0.39 & 0.03 & 0.43 & Eclipse_CplHist3 \\
\hline Ittoqqortoormiit & -0.02 & 0.03 & -0.26 & 0.35 & 0.03 & 0.01 & CMIP6_Cpl_Hist \\
\hline Kangerlussuaq & 0.03 & 0.05 & 0.37 & 0.64 & 0.09 & 0.50 & Eclipse_AMIP \\
\hline Kangerlussuaq & 0.01 & 0.04 & 0.08 & 0.49 & 0.05 & 0.22 & Eclipse_AMIP_NCEP \\
\hline Kangerlussuaq & -0.02 & 0.03 & -0.32 & 0.36 & 0.03 & 0.04 & Eclipse_CplHist1 \\
\hline Kangerlussuaq & -0.02 & 0.02 & -0.24 & 0.29 & 0.03 & 0.35 & Eclipse_CplHist2 \\
\hline Kangerlussuaq & -0.01 & 0.03 & -0.07 & 0.41 & 0.04 & 0.40 & Eclipse_CplHist3 \\
\hline Kangerlussuaq & 0.00 & 0.03 & -0.05 & 0.38 & 0.03 & 0.06 & CMIP6_Cpl_Hist \\
\hline Kuopio & -0.04 & 0.04 & -0.41 & 0.44 & 0.05 & -0.19 & Eclipse_AMIP \\
\hline Kuopio & -0.04 & 0.04 & -0.43 & 0.46 & 0.05 & -0.18 & Eclipse_AMIP_NCEP \\
\hline Kuopio & 0.04 & -0.46 & 0.46 & 0.05 & -0.16 & Eclipse_CplHist1 \\
\hline Kuopio & -0.40 & 0.41 & 0.05 & 0.23 & Eclipse_CplHist2 \\
\hline
\end{tabular}




\begin{tabular}{|l|c|c|c|c|c|c|l|}
\hline Kuopio & -0.04 & 0.04 & -0.40 & 0.41 & 0.05 & -0.06 & Eclipse_CplHist3 \\
\hline Kuopio & -0.04 & 0.04 & -0.40 & 0.41 & 0.05 & 0.10 & CMIP6_Cpl_Hist \\
\hline Narsarsuaq & -0.01 & 0.02 & -0.10 & 0.24 & 0.02 & -0.22 & Eclipse_AMIP \\
\hline Narsarsuaq & 0.00 & 0.02 & -0.03 & 0.30 & 0.03 & -0.18 & Eclipse_AMIP_NCEP \\
\hline Narsarsuaq & -0.02 & 0.03 & -0.23 & 0.35 & 0.03 & -0.99 & Eclipse_CplHist1 \\
\hline Narsarsuaq & -0.01 & 0.02 & -0.14 & 0.23 & 0.02 & -0.79 & Eclipse_CplHist2 \\
\hline Narsarsuaq & -0.02 & 0.02 & -0.33 & 0.33 & 0.03 & -0.15 & Eclipse_CplHist3 \\
\hline Narsarsuaq & 0.02 & 0.04 & 0.34 & 0.52 & 0.06 & -0.03 & CMIP6_Cp1_Hist \\
\hline OPAL & -0.03 & 0.03 & -0.41 & 0.45 & 0.04 & 0.57 & Eclipse_AMIP \\
\hline OPAL & -0.03 & 0.03 & -0.44 & 0.46 & 0.04 & 0.30 & Eclipse_AMIP_NCEP \\
\hline OPAL & -0.03 & 0.04 & -0.47 & 0.57 & 0.05 & -0.39 & Eclipse_CplHist1 \\
\hline OPAL & -0.03 & 0.04 & -0.44 & 0.49 & 0.04 & 0.23 & Eclipse_CplHist2 \\
\hline OPAL & -0.03 & 0.04 & -0.45 & 0.50 & 0.04 & 0.18 & Eclipse_CplHist3 \\
\hline OPAL & -0.02 & 0.04 & -0.29 & 0.56 & 0.05 & -0.69 & CMIP6_Cpl_Hist \\
\hline PEARL & -0.03 & 0.03 & -0.41 & 0.42 & 0.04 & 0.54 & Eclipse_AMIP \\
\hline PEARL & -0.03 & 0.04 & -0.43 & 0.51 & 0.05 & 0.04 & Eclipse_AMIP_NCEP \\
\hline PEARL & -0.03 & 0.04 & -0.47 & 0.50 & 0.05 & -0.01 & Eclipse_CplHist1 \\
\hline PEARL & -0.03 & 0.03 & -0.44 & 0.46 & 0.04 & 0.19 & Eclipse_CplHist2 \\
\hline PEARL & -0.03 & 0.03 & -0.35 & 0.42 & 0.04 & 0.12 & Eclipse_CplHist3 \\
\hline PEARL & -0.03 & 0.04 & -0.35 & 0.49 & 0.05 & -0.28 & CMIP6_Cpl_Hist \\
\hline Resolute_Bay & -0.04 & 0.04 & -0.46 & 0.47 & 0.06 & 0.39 & Eclipse_AMIP \\
\hline Resolute_Bay & -0.43 & 0.43 & 0.05 & 0.56 & Eclipse_AMIP_NCEP \\
\hline Resolute_Bay & 0.50 & 0.06 & 0.24 & Eclipse_CplHist1 \\
\hline
\end{tabular}




\begin{tabular}{|l|c|c|c|c|c|l|l|}
\hline Resolute_Bay & -0.04 & 0.04 & -0.48 & 0.48 & 0.06 & 0.45 & Eclipse_CplHist2 \\
\hline Resolute_Bay & -0.04 & 0.04 & -0.45 & 0.47 & 0.05 & 0.48 & Eclipse_CplHist3 \\
\hline Resolute_Bay & -0.03 & 0.04 & -0.36 & 0.40 & 0.05 & 0.05 & CMIP6_Cpl_Hist \\
\hline Sodankyla & -0.03 & 0.05 & -0.35 & 0.55 & 0.05 & -0.60 & Eclipse_AMIP \\
\hline Sodankyla & -0.03 & 0.04 & -0.38 & 0.50 & 0.05 & -0.62 & Eclipse_AMIP_NCEP \\
\hline Sodankyla & -0.04 & 0.05 & -0.48 & 0.54 & 0.06 & -0.93 & Eclipse_CplHist1 \\
\hline Sodankyla & -0.03 & 0.06 & -0.36 & 0.68 & 0.06 & -0.81 & Eclipse_CplHist2 \\
\hline Sodankyla & -0.03 & 0.04 & -0.36 & 0.50 & 0.05 & -0.76 & Eclipse_CplHist3 \\
\hline Sodankyla & -0.03 & 0.04 & -0.40 & 0.45 & 0.05 & -0.29 & CMIP6_Cpl_Hist \\
\hline Thule & -0.04 & 0.04 & -0.43 & 0.49 & 0.05 & 0.07 & Eclipse_AMIP \\
\hline Thule & -0.04 & 0.04 & -0.47 & 0.51 & 0.05 & 0.36 & Eclipse_AMIP_NCEP \\
\hline Thule & -0.04 & 0.05 & -0.52 & 0.56 & 0.05 & 0.05 & Eclipse_CplHist1 \\
\hline Thule & -0.04 & 0.04 & -0.48 & 0.51 & 0.05 & 0.20 & Eclipse_CplHist2 \\
\hline Thule & -0.03 & 0.04 & -0.41 & 0.49 & 0.05 & 0.15 & Eclipse_CplHist3 \\
\hline Thule & -0.03 & 0.04 & -0.35 & 0.49 & 0.05 & -0.26 & CMIP6_Cp1_Hist \\
\hline Tiksi & -0.14 & 0.14 & -0.67 & 0.67 & 0.23 & 0.00 & Eclipse_AMIP \\
\hline Tiksi & -0.15 & 0.15 & -0.72 & 0.72 & 0.24 & -0.02 & Eclipse_AMIP_NCEP \\
\hline Tiksi & -0.16 & 0.16 & -0.78 & 0.78 & 0.24 & 0.30 & Eclipse_CplHist1 \\
\hline Tiksi & -0.16 & 0.16 & -0.76 & 0.76 & 0.24 & 0.03 & Eclipse_CplHist2 \\
\hline Tiksi & -0.14 & 0.14 & -0.68 & 0.68 & 0.23 & 0.00 & Eclipse_CplHist3 \\
\hline Tiksi & -0.15 & 0.15 & -0.74 & 0.74 & 0.23 & 0.67 & CMIP6_Cp1_Hist \\
\hline Yakutsk & -0.66 & 0.66 & 0.15 & 0.44 & Eclipse_AMIP \\
\hline Yakutsk & 0.56 & 0.14 & 0.45 & Eclipse_AMIP_NCEP \\
\hline
\end{tabular}




\begin{tabular}{|l|c|l|l|l|l|l|l|}
\hline Yakutsk & -0.12 & 0.12 & -0.60 & 0.62 & 0.15 & 0.38 & Eclipse_CplHist1 \\
\hline Yakutsk & -0.13 & 0.13 & -0.66 & 0.66 & 0.15 & 0.49 & Eclipse_CplHist2 \\
\hline Yakutsk & -0.12 & 0.12 & -0.65 & 0.65 & 0.15 & 0.34 & Eclipse_CplHist3 \\
\hline Yakutsk & -0.10 & 0.11 & -0.54 & 0.57 & 0.14 & 0.36 & CMIP6_Cp1_Hist \\
\hline Yellowknife_Aurora & -0.07 & 0.10 & -0.46 & 0.63 & 0.17 & -0.35 & Eclipse_AMIP \\
\hline Yellowknife_Aurora & -0.07 & 0.10 & -0.47 & 0.61 & 0.17 & -0.09 & Eclipse_AMIP_NCEP \\
\hline Yellowknife_Aurora & -0.10 & 0.10 & -0.62 & 0.62 & 0.18 & -0.27 & Eclipse_CplHist1 \\
\hline Yellowknife_Aurora & -0.08 & 0.09 & -0.51 & 0.60 & 0.16 & 0.14 & Eclipse_Cp1Hist2 \\
\hline Yellowknife_Aurora & -0.09 & 0.10 & -0.55 & 0.63 & 0.18 & -0.30 & Eclipse_CplHist3 \\
\hline Yellowknife_Aurora & -0.08 & 0.11 & -0.51 & 0.67 & 0.18 & -0.31 & CMIP6_Cp1_Hist \\
\hline
\end{tabular}



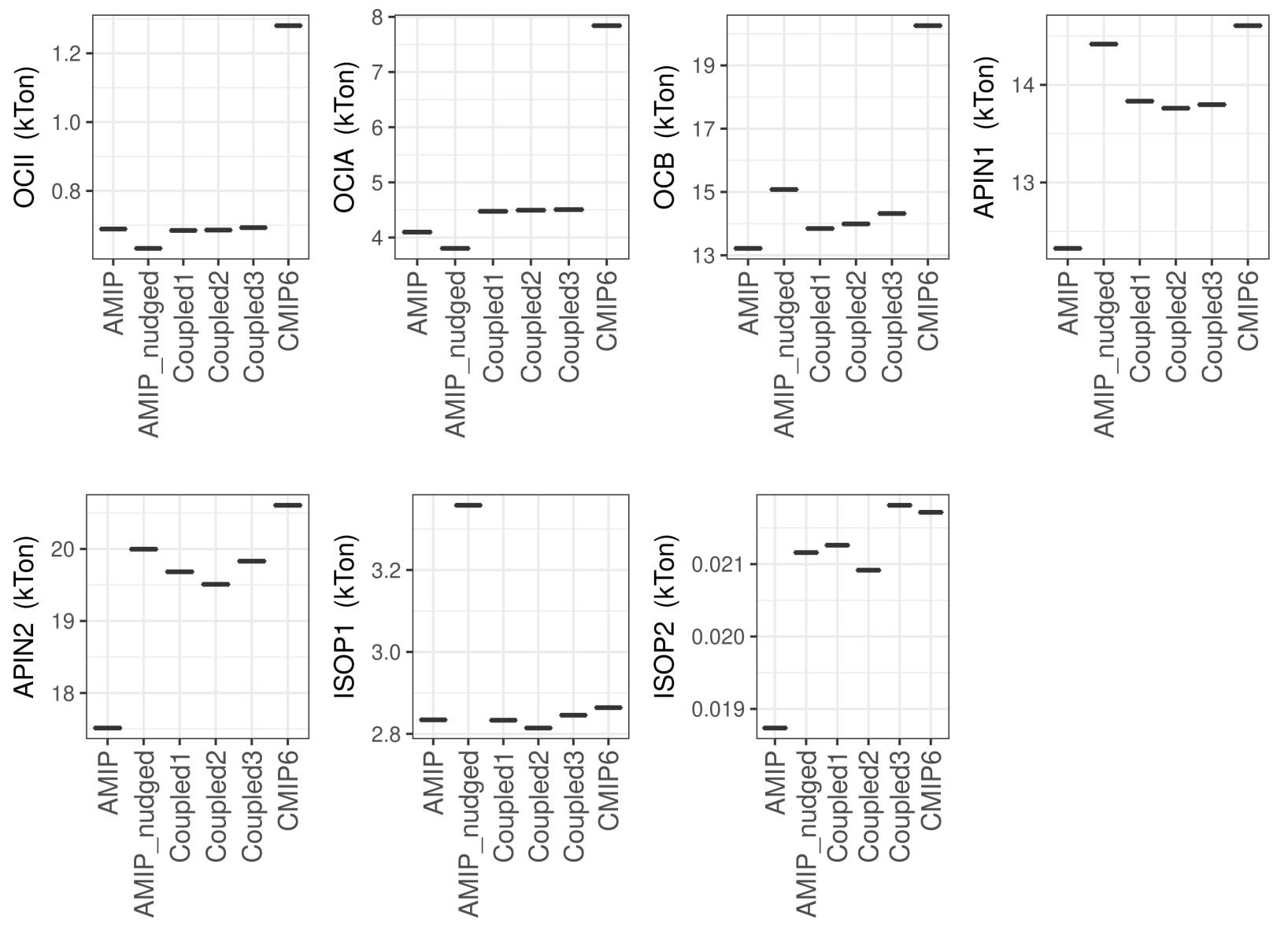

Figure S1. Present day SOA burdens in the Eclipse and CMIP6 ensemble. 


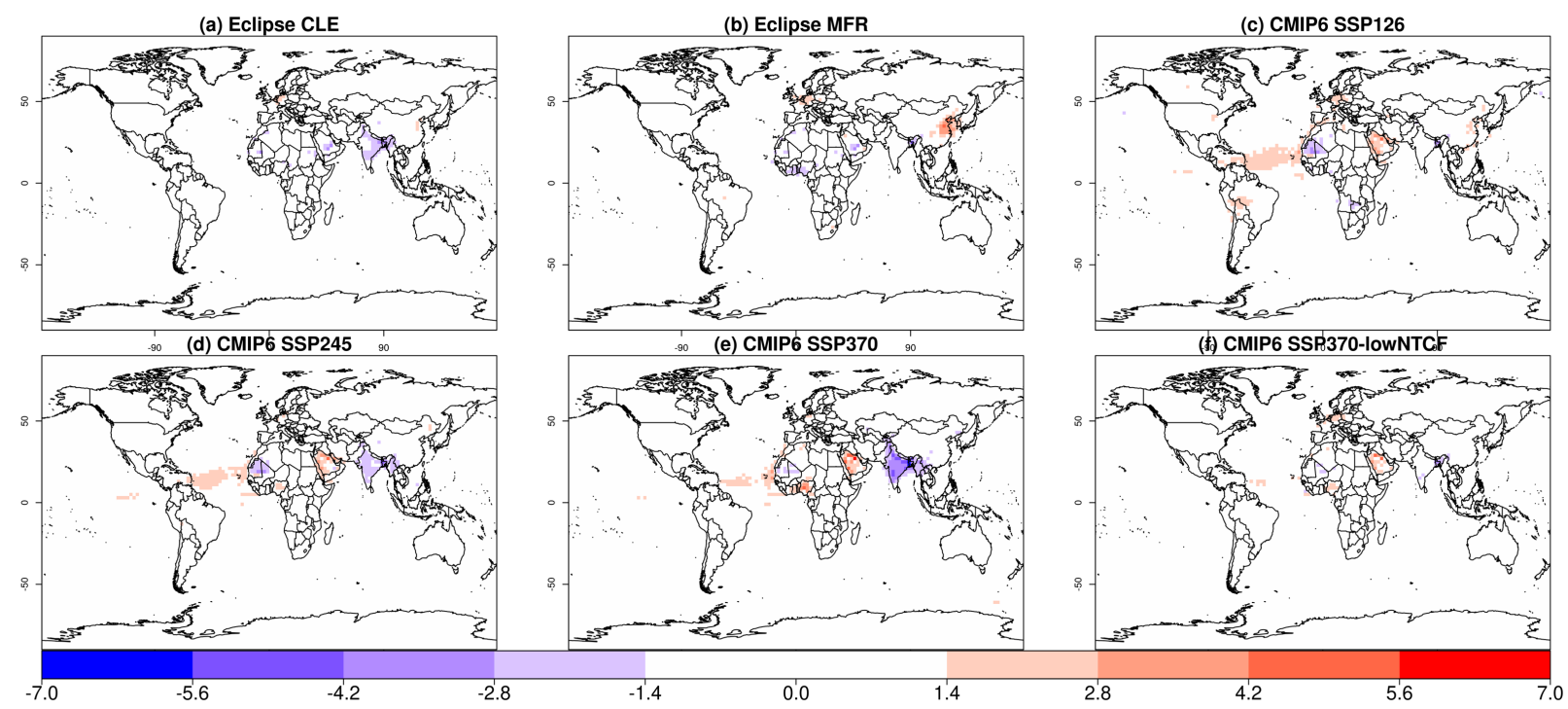

Figure S2. Spatial distribution of the statistically significant annual mean global $\mathrm{RF}_{\mathrm{ARI}}\left(\mathrm{W} \mathrm{m}^{-2}\right)$ changes between the 1990-2010 mean and the 2030-2050 mean as calculated by the GISS-E2.1 ensemble.

(a) Eclipse CLE

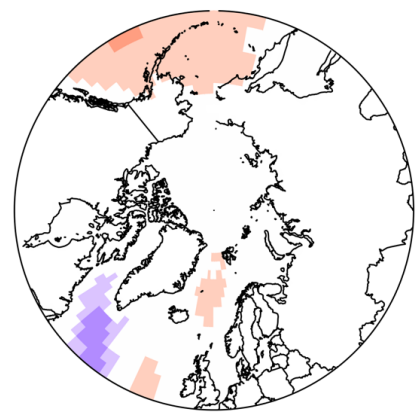

(d) CMIP6 SSP245

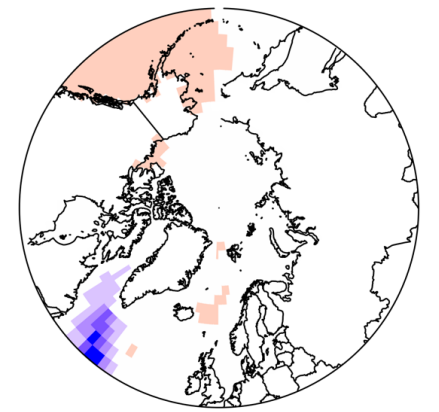

(b) Eclipse MFR

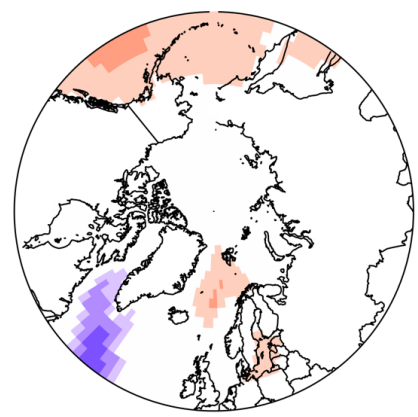

(e) CMIP6 SSP370

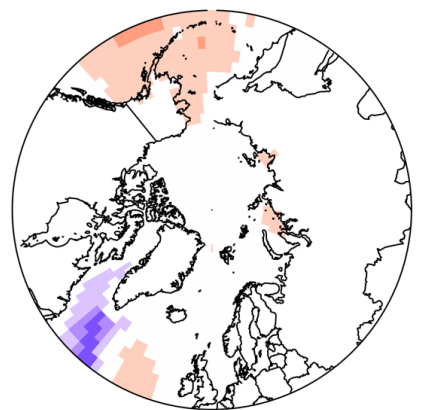

(c) CMIP6 SSP126

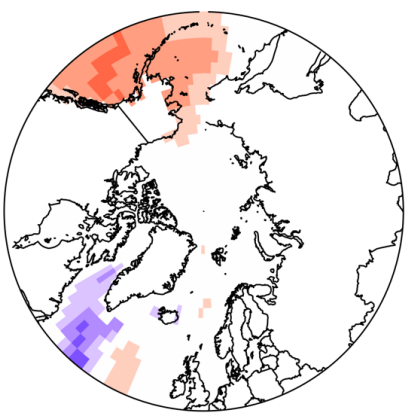

(f) CMIP6 SSP370-lowNTCF

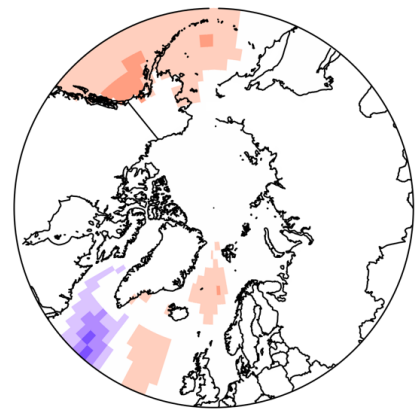

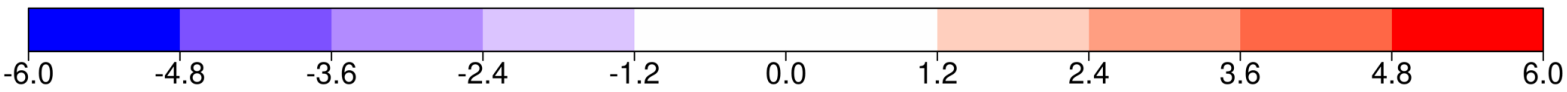

Figure S3. Spatial distribution of the annual mean Arctic sea surface temperature $\left({ }^{\circ} \mathrm{C}\right)$ change between the 1990-2010 mean and the 2030-2050 mean as calculated by the GISS-E2.1 ensemble. 
(a) Eclipse CLE

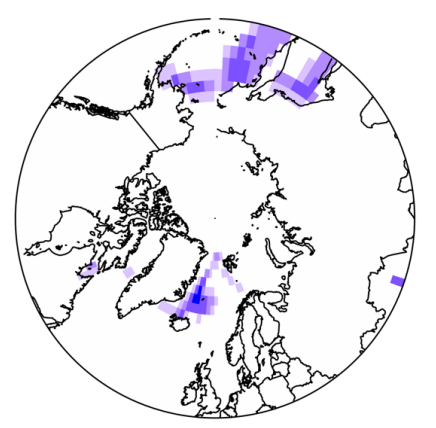

(d) CMIP6 SSP245

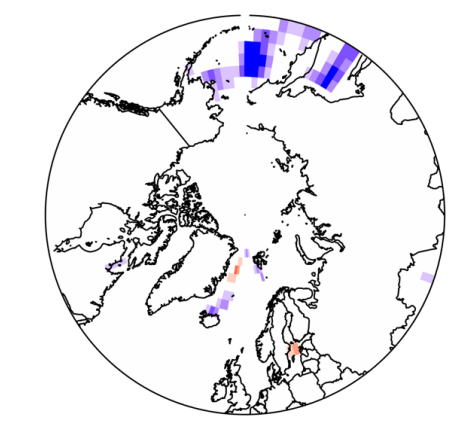

(b) Eclipse MFR

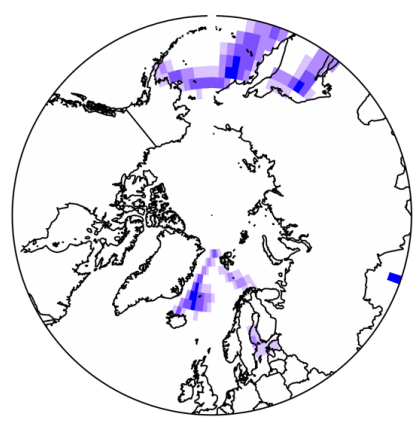

(e) CMIP6 SSP370

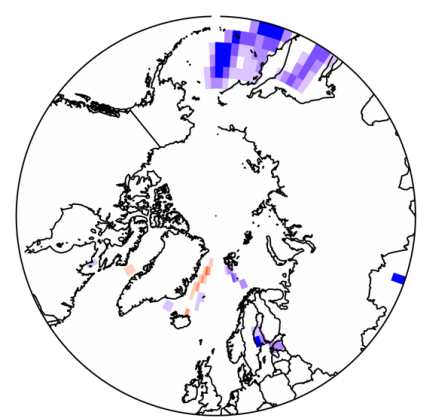

(c) CMIP6 SSP126

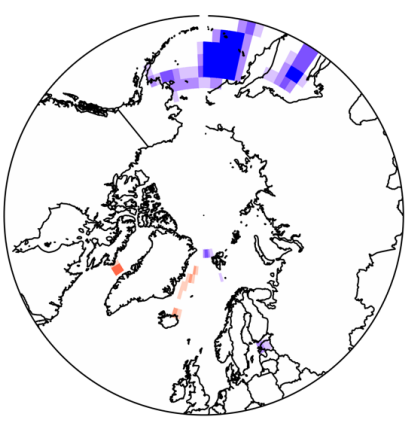

(f) CMIP6 SSP370-lowNTCF

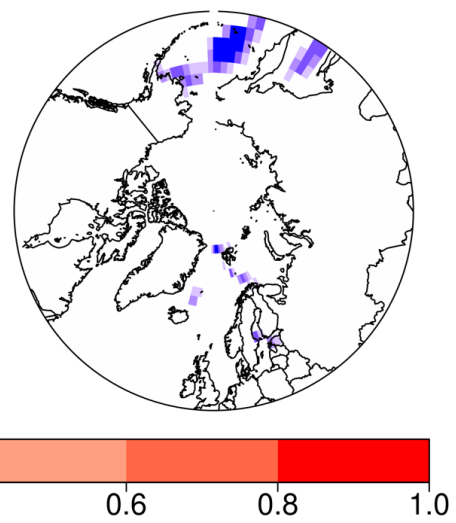

Figure S4. Spatial distribution of the March Arctic sea-ice fraction change between the 19902010 mean and the 2030-2050 mean as calculated by the GISS-E2.1 ensemble. 

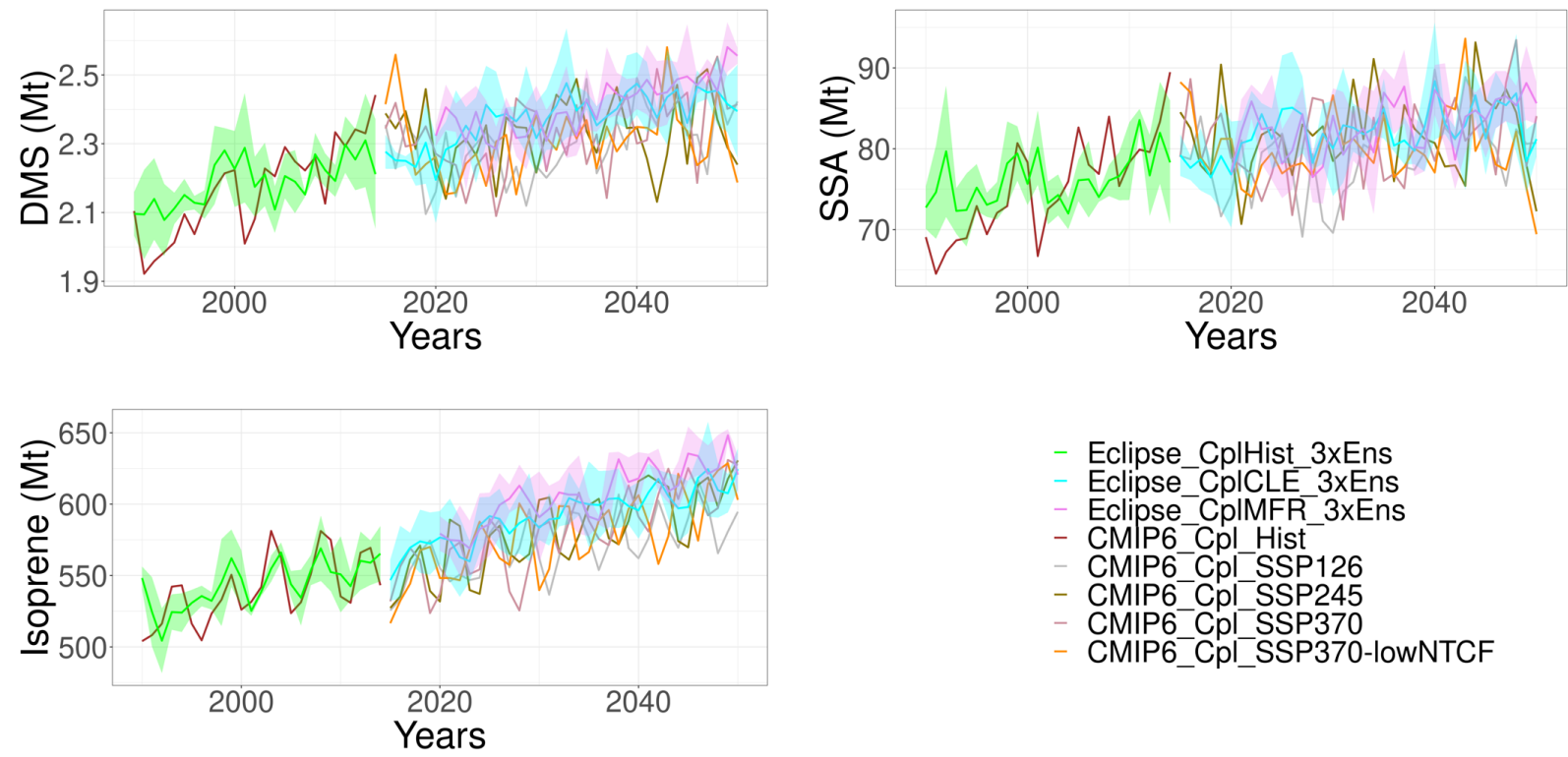

- Eclipse_CplHist_3xEns Eclipse CplCLE 3xEns - Eclipse CpIMFR 3xEns

- CMP6- Cpl Hist

- CMIP6-Cpl-SSP126

- CMIP6 $-\mathrm{Cpl}$ SSP245

- CMIP6_Cpl_SSP370

- CMIP6_Cpl_SSP370-lowNTCF

Figure S5. Arctic DMS and sea-salt and global isoprene emissions anomalies in 1900-2050 based on the 1990-2010 mean, simulated by the Eclipse and CMIP6 ensembles.

\section{References}

Bauguitte, S., Facility for airborne atmospheric measurements: Science instruments, Available at http://www.faam.ac.uk/index.php/science-instruments/chemistry/64-instruments , 2014.

Biraud, S. C., Carbon Monoxide Mixing Ratio System Handbook, U.S. Dept. of Energy, ARM Clim. Res. Facil., Washington, D. C., 2011.

Bottenheim, J.W., J.D. Fuentes, D.W. Tarasick and K.G. Anlauf (2002), Ozone in the Arctic lower troposphere during winter and spring 2000 (ALERT2000), Atmospheric Environment, 36, 2535-2544.

Chow, J. C., Watson, J. G., Pritchett, L. C., Pierson, W. R., Frazier, C. a. and Purcell, R. G.: The dri thermal/optical reflectance carbon analysis system: description, evaluation and applications in U.S. Air quality studies, Atmos. Environ. Part A. Gen. Top., 27(8), 11851201, doi:10.1016/0960-1686(93)90245-T, 1993

Betty Croft, R. V. Martin, W. Richard Leaitch, J Burkart, R.Y.-W. Chang, D. B. Collins, P. L. Hayes, A. L. Hodshire6, L. Huang, J. K. Kodros, A. Moravek4, E. L. Mungall, J. G. Murphy, S. Sharma, S. Tremblay, G. R. Wentworth, M. D. Willis, J. P. D. Abbatt, and J. R. Pierce, Arctic 
marine secondary organic aerosol contributes significantly to summertime particle size distributions in the Canadian Arctic Archipelago, Atmos. Chem. Phys., 19, 2787-2812, 2019, https://doi.org/10.5194/acp-19-2787-2019.

Dabek-Zlotorzynska, E., Dann, T. F., Martinelango, P. K., Celo, V. Brook, J. R., Mathieu, D., Ding, L., Austin, C. C., Canadian National Air Pollution Surveillance (NAPS) PM2.5 speciation program: Methodology and PM2.5 chemical composition for the years 2003-2008, Atmospheric Environment,45,3, 2011,673-686, https://doi.org/10.1016/j.atmosenv.2010.10.024.

EMEP manual, https://projects.nilu.no/ccc/manual// 2014.

Galbally, I.E., Schultz, M.G., Buchmann, B., Gilge, S., Guenther, F., Koide, H., Oltmans, S., Patrick, L., Scheel, H.-E., Smit, H., Steinbacher, M., Steinbrecht, W., Tarasova, O., Viallon, J., Volz-Thomas, A., Weber, M., Wielgosz R. and Zellweger C. Guidelines for Continuous Measurement of Ozone in the Troposphere, GAW Report No 209, Publication WMO-No. 1110, ISBN 978-92-63-11110-4, World Meteorological Organisation, Geneva Switzerland, 76 pp., 2013. http://www.wmo.int/pages/prog/arep/gaw/gaw-reports.html

Harris, D.: Quantitative Chemical Analysis, 6th ed., edited by M. L. Byrd, Michelle Russel Julet, New York., 2003.

Kondo, Y., L. Sahu, N. Moteki, F. Khan, N. Takegawa, X. Liu, M. Koike, T. Miyakawa (2011), Consistency and traceability of black carbon measurements made by laser-induced incandescence, thermal-optical transmittance, and filter-based photo-absorption techniques, Aerosol Sci. Tech., 45, 295-312, DOI: 10.1080/02786826.2010.533215.

Leaitch, W. R., Sharma,s., Huang, L., Toom-Sauntry, D., Chivulescu, A., Macdonald, A.A., von Salzen, K., Dimethyl Sulfide Control of the Clean Summertime Arctic Aerosol and Cloud, Elementa Science of the Anthropocene 1: 000017, 2013, doi:10.12952/journal.elementa.000017dlementascience.org

Malm, W. C., Sisler, J. F., Huffman, D., Eldred, R. A., and Cahill, T. A.: Spatial and seasonal trends in particle concentration and optical extinction in the United States, J. Geophys. Res., 99, 1347-1370, 1994.

Nattinger, Kristian C. Temporal and Spatial Trends of Fine Particulate Matter Composition in Fairbanks, Alaska, PhD thesis, University of Alaska, Fairbanks, 2016.

Sharma, S., W. R. Leaitch, L. Huang, Daniel Veber, Felicia Kolonjari, Wendy Zhang, Sarah J. Hanna, Allan K. Bertram, and John A. Ogren, An evaluation of three methods for measuring 
black carbon in Alert, Canada, Atmos. Chem. Phys., 17, 15225-15243, 2017, https://oi.org/10.5194/acp-17-15225-2017.

Sharma, S., Barrie, L.A., Magnusson, E., Brattstrom, G., Leaitch, W. R., Steffen, A., and Landsberger, S., A factor and trends analysis of multidecadal lower tropospheric observations of arctic aerosol composition, black carbon, ozone, and mercury at Alert, Canada. J.Geophys. Res.:Atmospheres, 124, 14,133-14,161, 2019, http://doi.org/10.1029/2019JD030844.

Skov, H. Christensen, J. Goodsite, M.E. Heidam, N.Z. Jensen, B. Wåhlin, P. and Geernaert, G.: The fate of elemental mercury in Arctic during atmospheric mercury depletion episodes and the load of atmospheric mercury to Arctic. ES \& T, 38, 2373-2382, 2004.

Tørseth, K., Aas, W., Breivik, K., Fjæraa, A. M., Fiebig, M., Hjellbrekke, A. G., Lund Myhre, C., Solberg, S., and Yttri, K. E.: Introduction to the European Monitoring and Evaluation Programme (EMEP) and observed atmospheric composition change during 1972-2009, Atmos. Chem. Phys., 12, 5447-5481, doi:10.5194/acp-12-5447-2012, 2012. 